\title{
ResearchOnline@JCU
}

This is the author-created version of the following work:

Heng, Audrey, Heal, Clare, Banks, Jennifer, and Preston, Robyn (2018) Transgender peoples' experiences and perspectives about general healthcare: a systematic review. International Journal of Transgenderism, 19 (4) pp. 359-378.

Access to this file is available from:

https://researchonline.jcu.edu.au/54712/

Please refer to the original source for the final version of this work: 
Transgender Peoples’ Experiences and Perspectives About General Healthcare:

A Systematic Review

Audrey Heng ${ }^{1}$, Professor Clare Heal ${ }^{1}$, Dr Jennifer Banks ${ }^{1}$, Dr Robyn Preston ${ }^{1}$ College of Medicine and Dentistry, James Cook University

Funding: This review was not funded.

Conflict of Interest: The authors declare that they have no conflict of interest.

Ethical Considerations: This article does not contain any studies with human participants performed by any of the authors. 


\section{Transgender Peoples’ Experiences and Perspectives About General Healthcare:}

\section{A Systematic Review}

Background: Healthcare plays a vital role in the health and quality of life of transgender people, particularly those who wish to medically and/or surgically transition. In these cases, healthcare experiences can have a significant impact on the transgender person's perception of healthcare and future interactions with the healthcare system. Aim: To explore and systematically review the literature related to transgender peoples' experiences and perspectives of general healthcare. Methods: A systematic search of MEDLINE, CINAHL, Scopus, Web of Science, Informit and PsycINFO was performed according to established search strategies. Results were screened for eligibility in two parts. Titles and abstracts were screened for relevance, and then full-texts were retrieved and screened using eligibility criteria, resulting in 20 articles of primary research included in the review. Data was extracted from the studies and thematically analysed to synthesise findings. Results: Four main themes were identified relating to healthcare provider (HCP) knowledge, HCP communication, relationship dynamics, and healthcare systems. These reflected a subtheme of perceived underlying cisnormativity and transphobia which influenced transgender patients' interactions with the healthcare system. Participants also provided suggestions for improved care experiences. Discussion: Findings from prior studies and reviews about other transgender healthcare related topics describe similar themes and recommendations from participants. This reflects an impetus towards shared dialogue and compromise between HCPs, policy makers and transgender patients to listen to each other's experiences and work collaboratively to improve transgender healthcare. Further research is necessary to explore and target geographic, demographic, and specific healthcare aspects regarding potential implementation of these recommendations.

Keywords: transgender, healthcare, experiences, perspectives 


\section{Transgender Peoples’ Experiences and Perspectives About General Healthcare:}

\section{A Systematic Review}

Gender and sex are often used as interchangeable terms, however this creates confusion where gender diverse people are concerned. Gender refers to the sociocultural perception and delineation of masculinity and femininity, or man and woman. Sex refers to the biological classification of male and female (Nobelius, 2004). Gender diversity refers to variations in gender beyond the binary of man and woman (American Psychological Association, 2015), and for the purpose of this review will refer to non-cisgender individuals. ${ }^{1}$ Transgender, or trans, people - the focus of this review - are just one subset of gender diversity. When a person is trans their gender does not match their sex assigned at birth (GLAAD, n.d.).

It has been frequently observed that trans people are amalgamated with their sexual minority counterparts in research and literature. However, the trans population faces unique health issues including those related to transition (social, medical and surgical processes associated with alignment of physical sex to reflect gender identity), and poorer mental health than both the general population and their non-heterosexual cisgender counterparts. According to a 2013 Australian report by Rosenstreich, trans people experience higher rates of mood disorders, are subjected to increased levels of almost all types of violence, and have higher rates of suicidal ideation and suicide attempts. This is supported by a study from the United Kingdom (UK) where 84\% of trans participants had considered ending their lives at some point, (McNeil, Bailey, Ellis, Morton, \& Regan, 2012) compared to approximately one-

\footnotetext{
${ }^{1}$ It should be noted that many definitions exist for both "gender diversity" and "transgender" and these are often evolving. Therefore, given the individual's definition of the terms, not all trans people identify as gender diverse; e.g. someone assigned male at birth may identify as a binary woman and not as gender diverse. Similarly, not all gender diverse individuals identify as trans.
} 
fifth of the general UK population over the course of a lifetime (Sinclair \& Leach, 2017). The aforementioned issues have been linked to the experience of or fear of discrimination, exclusion, and isolation (Rosenstreich, 2013). Additionally, studies from the United States (US) and UK highlight a correlation between perceived stigma and discrimination, and poorer health of trans people (Ellis, Bailey, \& McNeil, 2015; White Hughto, Reisner, \& Pachankis, 2015).

With increasing discussion about trans people in the media - from high-profile celebrities to debates about pronouns and bathrooms - the potential likelihood of exposure to transphobic beliefs rises. Therefore, the need to access healthcare may also increase in order to address resultant poor health. For trans people intending to medically and/or surgically transition, encounters with the healthcare system can be imperative to achieving good quality of life (McNeil et al., 2012), and accessing safe medical and surgical interventions by minimising non-prescribed hormone use and self-performed surgeries (de Haan, Santos, Arayasirikul, \& Raymond, 2015; Rotondi et al., 2013). The perspectives of trans people regarding their experiences with general healthcare are therefore vital to ensure healthcare delivery is sensitive to the unique needs of this population and minimises unintentional harm.

Prior reviews have examined the mental health needs of trans people, and the barriers and facilitators to this population utilising healthcare (Lerner \& Robles, 2017; McCann \& Sharek, 2016). However, to the author's knowledge, no reviews address transgender peoples’ experiences and perspectives of general healthcare. The purpose of this review, therefore, is to explore the perspectives of trans people regarding their experiences with general healthcare. A systematic review was conducted which examined and described the literature addressing the research question, "What are the experiences and/or perspectives that transgender people have about their general healthcare?” 


\section{Methods}

\section{Protocol and Registration}

The review protocol was registered with PROSPERO (CRD42017055738). This is available at

http://www.crd.york.ac.uk/PROSPERO/display_record.asp?ID=CRD42017055738.

\section{Search Methods}

An initial search of MEDLINE and CINAHL defined the scope of the study and finalised relevant search terms contained in the title, abstract or keywords of articles. A systematic search of relevant studies was performed in the following databases: MEDLINE, CINAHL, Informit, PsycINFO, Scopus, and Web of Science. Keywords and MeSH terms reflected the concepts of "transgender," "healthcare” and "perspectives.” Database-specific search strategies, including specific keywords, subject headings and Boolean operators, can be found in the Appendix. If available, the following limitations were applied to the search results: humans, English language, and peer-reviewed sources. The data retrieved was dated from subject inception to April 2018. The last search was performed on April 1st, 2018.

\section{Inclusion and Exclusion Criteria}

The inclusion and exclusion criteria were:

- Transgender or transsexual experiences and/or perspectives:

o Include: trans-identifying non-binary or gender non-conforming persons.

o Exclude: lesbian, gay, bisexual and transgender (LGBT) community; cisgender sexual minorities; men who have sex with men (MSM); healthcare providers; co-occurring conditions (i.e. trans + another health issue).

- Receiving of or interactions with general healthcare only: 
o Include: primary care, trans-specific care, general healthcare, multiple specialities.

o Exclude: specific healthcare focus (e.g. mental health, emergency department, HIV); social services; access to care.

- Literature type:

o Include: quantitative, qualitative and mixed methods primary research articles.

o Exclude: opinion pieces, feature articles, editorials, individual case reports, reviews, books.

\section{Study Selection}

Twenty (20) studies were eligible for inclusion in the review. Figure 1 demonstrates the screening process. Data was extracted from the 20 studies and study characteristics were summarised under the following headings: year, country, participant group, ages, sample size or participant number, healthcare focus, method, and key findings (Table 1).

\section{Quality Assessment}

The 20 eligible studies were critically appraised using a rubric adapted from the criteria and scoring system developed by Pluye, Gagnon, Griffiths, \& Johnson-Lafleur (2009) for mixed methods studies. The associated scoring system was not utilised as it implies equal weight of all criteria. Table 2 provides the descriptive assessment for individual studies included in this review. The appraisal criteria in Table 3 allocates studies to the standard of “Poor," "Satisfactory,” or “Good.” Table 4 provides visual summary of the quality appraisal. 


\section{Results}

\section{Overview of Studies}

Twenty (20) studies fulfilled the inclusion criteria. Data was extracted and organised by study characteristics (Table 1) with included studies published between 2008 and 2018. All studies included trans participants, but preferred terms participants used to describe their gender identity varied (e.g. gender diverse, genderqueer). Three studies included data solely from male-identifying trans people (trans men); two of these specifically focused on trans men, while one study was still ongoing but all existing participants were trans men. All studies were based in Western countries, most commonly focusing on the US ( $n=9)$, followed by Canada ( $\mathrm{n}=4)$ and Sweden $(\mathrm{n}=4)$. The remaining studies focused on Brazil, Australia, and Australia and New Zealand together. Participants were predominantly transgender adults, although some studies included adolescents. The lowest age reported was 13 years and the highest age reported was 74 years, however some studies only provided a mean, minimum or maximum age, and one study did not provide any age data.

Study methods included quantitative $(n=3)$, qualitative $(n=12)$, and mixed methods $(n=5)$. Qualitative research methods were predominantly interviews (in-depth and semistructured), but also included participant observation and focus groups. Participant numbers ranged from 3 to 85 participants. All quantitative studies were cross-sectional surveys, with one collecting primary data from 433 participants, another from 626 participants, and the other using existing data from 1,711 transmen who completed the National Transgender Discrimination Survey. Of the mixed methods studies, three utilised a survey design for data collection with qualitative analysis of responses to open-ended questions included in the survey. The remaining mixed methods studies had a qualitative component using focus groups or semi-structured interviews to collect participant experiences, but used a 
questionnaire to collect demographic information. Mixed methods participant numbers ranged from 7 to 253 participants.

Most studies achieved an appraisal level to an overall satisfactory or good standard. Studies were considered good if all criteria met the requirements of a good standard. Studies were considered poor if requirements of more than one criteria were poorly met. All other studies were considered satisfactory. Most qualitative studies were appraised as satisfactory or good, with 5 of the 12 studies considered good overall (Hudson, 2018; Linander, Alm, Hammarström, \& Harryson, 2017; Poteat, German, \& Kerrigan, 2013; Roller, Sedlak, \& Draucker, 2015; Ross \& Bell, 2017). Studies rated as poor or satisfactory failed to discuss researchers' reflexivity to a satisfactory standard; however, this was the only criteria where studies rated as satisfactory did not meet the criteria for good quality (Bauer et al., 2009; Lindroth, 2016; Taylor, 2013; Vermeir, Jackson, \& Marshall, 2018; von Vogelsang, Milton, Ericsson, \& Strömberg, 2016; Westerbotn et al., 2017). Two quantitative studies were appraised as good (Bauer, Zong, Scheim, Hammond, \& Thind, 2015; Shires \& Jaffee, 2015). Costa et al. (2018) was appraised as satisfactory overall. Appraisal of the mixed methods studies found these were of varying quality. All mixed methods studies failed to discuss researchers’ reflexivity similar to many of the qualitative studies. Riggs, Coleman, \& Due (2014) was highest rated of the included mixed methods studies, meeting requirements of all other criteria to be rated as of good quality. Of the 5 mixed methods studies, 3 failed to address the quantitative aspects of their study to a satisfactory standard, and did not clearly justify their mixed methods approach (Guss et al., 2017; Hinrichs et al., 2018; Pitts, Couch, Mulcare, Croy, \& Mitchell, 2009).

The areas of healthcare addressed by the studies ranged from a single focus (e.g. family practice) to multi-focus (e.g. general practice, surgery and psychiatry) and/or general healthcare, which Roller et al. (2015) described as “any aspects of participants’ healthcare 
experiences.” (p. 419) Primary care, general practice and family practice were commonly mentioned. Trans-specific healthcare was also frequently mentioned and included gender identity clinics, psychiatry, surgery, and endocrinology, among other specialties. Due to the broad healthcare contexts described in the studies, and as some studies did not specify any particular healthcare context, the themes synthesised in this review have not been stratified to any particular healthcare field or specialty.

From the 20 included studies, descriptive coding identified four main themes, one subtheme, and two peripheral themes relating to trans peoples' experience and perception of healthcare. These were healthcare provider (HCP) knowledge, HCP communication, relationship dynamics, and healthcare systems. The first two themes were each discussed in 18 studies, and both themes occurred in 15 studies. The latter two themes occurred less frequently. Eight studies attributed participants’ experiences with these themes to the subtheme of underlying cisnormativity and transphobia, and 12 studies showed the choices participants made when navigating their healthcare experiences. In six studies, participants provided recommendations for improvement of the trans healthcare experience. These themes, subtheme, and peripheral themes are described below, and the relationship between them is depicted in Figure 2.

\section{Main Themes}

Healthcare provider knowledge. Healthcare provider (HCP) knowledge and education was a strongly recurring theme in 18 of the 20 included studies (Bauer et al., 2009; Bauer et al., 2015; Costa et al., 2018; Dewey, 2008; Guss et al., 2017; Hinrichs et al., 2018; Hudson, 2018; Linander et al., 2017; Lindroth, 2016; Pitts et al., 2009; Poteat et al., 2013; Riggs et al., 2014; Roller et al., 2015; Shires \& Jaffee, 2015; Taylor, 2013; Vermeir et al., 2018; von Vogelsang et al., 2016; Westerbotn et al., 2017). Participants described 
experiences that suggested a lack of HCP knowledge and education ranging from blatantly honest disclosure of limited knowledge, to subtler displays demonstrated through perceived attitudes of the HCP to the trans client. In Bauer et al.’s 2015 survey, 53.6\% of respondents were told their HCP did not know enough about trans-related care to provide care. In Bauer et al. (2009), participants recalled interactions where the trans patient was implied to be irrelevant or unimportant due to the rarity of their population group. Tied closely with this was perceived HCP ignorance and apparent expectations for the trans patient to fit a particular gender stereotype aligning to their identified gender in order to access transitionrelated care and avoid dispute about the patient's ‘choice’ of gender identity (Lindroth, 2016; Pitts et al., 2009; Taylor, 2013; von Vogelsang et al., 2016; Westerbotn et al., 2017).

Resulting from this perceived or actual lack of HCP knowledge, trans participants described educating their provider about trans people and healthcare. In multiple studies, participants claimed to be the source of medical information guiding their HCP in transitionrelated treatment plans, researching through trans community networks and internet sources (Costa et al., 2018; Dewey, 2008; Lindroth, 2016; Poteat et al., 2013; Riggs et al., 2014; Roller et al., 2015; Taylor, 2013, Vermeir et al., 2018). Trans participants also described encounters where they were asked many exploratory questions about trans people and the trans population; some expressed this to be "tiring” (von Vogelsang et al., 2016, p. 3582) and felt like “live teaching material” (Lindroth, 2016, p. 3516), while others appreciated the willingness of their HCP to learn about trans people and their needs (Riggs et al., 2014) and acknowledge their ignorance when unknowledgeable about topics necessary for adequate care (Westerbotn et al., 2017).

Healthcare provider knowledge experiences were perceived to be positive when the HCP was perceived to be knowledgeable or well-read about the trans population and their needs (Bauer et al., 2015; Pitts et al., 2009; Riggs et al., 2014). 
Healthcare provider communication. This theme was also present in 18 of the 20 reviewed studies (Bauer et al., 2009; Bauer et al., 2015; Costa et al., 2018; Dewey, 2008; Guss et al., 2017; Hinrichs et al., 2018; Hudson, 2018; Kosenko, Rintamaki, Raney, \& Maness, 2013; Lindroth, 2016; Pitts et al., 2009; Poteat et al., 2013; Riggs et al., 2014; Ross \& Bell, 2017; Shires \& Jaffee, 2015; Taylor, 2013; Vermeir et al., 2018; von Vogelsang et al., 2016; Westerbotn et al., 2017). The main sub-themes within HCP communication were related to the perceived acceptance or denial of the trans patient's identity and/or the professionalism displayed.

Trans patients recounted good experiences related to the apparent acceptance of, respect for, and/or affirmation of their identity as a trans person. This was demonstrated by the normalisation of transgenderism and being non-judgemental (Hinrichs et al., 2018; Hudson, 2018; Pitts et al., 2009; von Vogelsang et al., 2016). One participant in Hinrichs et al. (2018) “... really appreciate[s] when people can see me as a whole person. Like being trans is a part of me, but [...] there's a lot of other parts of me that come into play” (p. 79). The HCP's use of the patient's preferred pronouns and name was a key expression of identity affirmation. Trans participants also greatly appreciated the change of medical documentation to reflect or indicate their gender (Bauer et al., 2009; Guss et al., 2017; Hudson, 2018; Pitts et al., 2009; Riggs et al., 2014; Westerbotn et al., 2017). This, and perceived HCP comfort with the patient's gender identity, fostered a sense of trust, safety and confidence in the HCP (Guss et al., 2017; Hudson, 2018; Riggs et al., 2014; von Vogelsang et al., 2016); a participant in von Vogelsang et al.’s 2016 study even described re-evaluating HCPs in light of their previous negative experiences.

Experiences where the patient's trans identity was perceived to be denied by the HCP were the most negative, however overvaluation or undervaluation of the trans aspect of the patient's identity were also recounted as negative by participants (Bauer et al., 2009; Dewey, 
2008; Hudson, 2018; Lindroth, 2016; Ross \& Bell, 2017; Vermeir et al., 2018; Westerbotn et al., 2017). Denial of identity was felt by the use of insensitive language and terms when referring to trans people, and the use of incorrect pronouns or the patient's birth name (Bauer et al., 2015; Guss et al., 2017; Kosenko et al., 2013; Pitts et al., 2009; Ross \& Bell, 2017; Taylor, 2013; von Vogelsang et al., 2016). Participants also perceived HCP discomfort, uncertainty and awkwardness as denial of the trans identity (Bauer et al., 2009; Dewey, 2008; Guss et al., 2017; Hudson, 2018; Kosenko et al., 2013; Pitts et al., 2009; Poteat et al., 2013). Overvaluation of the trans aspect of identity was perceived by experiences of HCPs attributing any health issue to the patient's trans identity, even if the reason for seeking healthcare was not trans-related (Bauer et al., 2009; Ross \& Bell, 2017; Westerbotn et al., 2017). Undervaluation was perceived in encounters where the HCP completely ignored the trans aspect of the patient (Bauer et al., 2009; Dewey, 2008; Vermeir et al., 2018). As a result, trans patients described separating their gender identity from their medical problem and/or seeking healthcare from a source separate to the provider of their trans-specific care (Bauer et al., 2009; Hudson, 2018; Lindroth, 2016).

The use of correct pronouns and preferred name was closely linked to a sub-theme of perceived HCP professionalism. Professionalism was linked to sensitivity, respect, and responsiveness. Negative experiences were largely related to perceived or actual disrespect and mistreatment (Bauer et al., 2009; Costa et al., 2018; Hinrichs et al., 2018; Hudson, 2018; Kosenko et al., 2013; Lindroth, 2016; Pitts et al., 2009; Riggs et al., 2014; Ross \& Bell, 2017; Taylor, 2013; Vermeir et al., 2018; Westerbotn et al., 2017). In Kosenko et al.’s 2013 study, $71 \%$ of participants reported at least one instance of mistreatment, while in Costa et al.'s 2018 study, approximately 60\% of participants reported being discriminated against by HCPs in any situation. Blatant displays of disrespect and mistreatment included instances of verbal and physical abuse, and/or refusal of services with or without reason. One participant in Pitts 
et al. (2009) was told to “find god not hormones” (p. 488); a participant in Ross \& Bell (2017) shared a similar experience. Participants perceived rough physical examination technique, insufficient disclosure of medical information (e.g. adverse effects), and confidentiality breaches (accidental or deliberate) as experiences of "substandard care” (Kosenko et al., 2013, p. 820) or mistreatment due to disrespect (Hudson, 2018; Riggs et al., 2014; von Vogelsang et al., 2016). Comparatively, good professionalism was deemed apparent when the HCP appeared to be sensitive, respectful and responsive (Guss et al., 2017; Pitts et al., 2009; Riggs et al., 2014; von Vogelsang et al., 2016; Westerbotn et al., 2017). Sensitivity was displayed by acknowledgement and measures to reduce discomfort with physical examinations, the use of neutral language for gendered anatomy, the upholding of privacy and confidentiality in physical examinations and medical documentation, and being thoroughly informative regarding treatments or treatment options (Guss et al., 2017; Pitts et al., 2009; Riggs et al., 2014; von Vogelsang et al., 2016). Respect and responsiveness were described as the HCP putting effort into good communication with the patient such as listening, showing empathy, compassion and understanding, treating the patient as a person and focusing on the patient's needs, and being cooperative with the patient such as involving them in decision-making and their care (Hinrichs et al., 2018; Hudson, 2018; Pitts et al., 2009; Riggs et al., 2014; von Vogelsang et al., 2016; Westerbotn et al., 2017). Being perceived as willing to work with the trans patient was an important aspect of professionalism that had significant impact on the provider-patient relationship, elaborated below in its own theme.

Relationship dynamics. This theme had two key sub-themes related to power and support. Nine studies discussed perceived provider-patient power imbalance (Bauer et al., 2015; Dewey, 2008; Guss et al., 2017; Linander et al., 2017; Lindroth, 2016; Poteat et al., 2013; Riggs et al., 2014; Taylor, 2013; von Vogelsang et al., 2016). Participants expressed 
feeling dependent and vulnerable to their HCP, perceiving the HCP as holding a “gatekeeping” (Linander et al., 2017, p. 9; Riggs et al., 2014, p. 3) or evaluating role for the patient accessing transition-related care (Bauer et al., 2015; Dewey, 2008; Lindroth, 2016; Taylor, 2013; von Vogelsang et al., 2016). One participant from Dewey (2008) stated, "Until you are diagnosed as a transsexual you are a sexual deviant” (p. 1351). Additionally, some participants perceived instances of HCPs reinforcing and re-establishing their authority through displays of knowledge and doubt about the patient's healthcare knowledge (Bauer et al., 2015; Linander et al., 2017; Poteat et al., 2013). Occasions where patients were allegedly forced to undergo care (e.g. physical examinations, mental health services) or reveal unrelated private matters such as sexual behaviour were also perceived to reinforce the apparent power imbalance (Kosenko et al., 2013; von Vogelsang et al., 2016). Additionally, insufficient disclosure of information (e.g. side effects) by HCPs was perceived by some participants as withholding information, an act designed to reinforce HCP power (Riggs et al., 2014; von Vogelsang et al., 2016). Regarding the sub-theme of support, participants voiced feeling a lack of support throughout their healthcare encounters. This was felt through multiple aspects of their healthcare journey such as having a lack of psychosocial support during the waiting process for transition, a lack of support and follow up after receiving their desired treatment, and a lack of support from their HCP when accessing care specific to their birth sex (Linander et al., 2017; Lindroth, 2016; von Vogelsang et al., 2016).

Healthcare provider interactions were perceived as positive when participants felt they had a partnership with their HCP and held a role in the healthcare encounter (Hinrichs et al., 2018; Pitts et al., 2009; von Vogelsang et al., 2016), as exemplified by a participant in Hinrichs et al. (2018) - “[...] we’re a team. I really value that we are totally equal partners in what we're doing” (p. 79). This also helped foster a sense of support. Participants also described appreciating instances where their HCP helped to find loopholes and ambiguities in 
treatment indications or medical insurance claims and subsidies in order to negotiate systemic barriers which may otherwise exclude the trans person from care or result in increased financial burden (Lindroth, 2016; Roller et al., 2015). These systemic barriers will be stated below.

Healthcare systems. This theme was present in 11 studies (Bauer et al., 2009; Costa et al., 2018; Dewey, 2008; Hinrichs et al., 2018; Linander et al., 2017; Lindroth, 2016; Pitts et al., 2009; Roller et al., 2015; Ross \& Bell, 2017; Taylor, 2013; Vermeir et al., 2018). While the systemic and institutional issues mentioned by the trans participants are highly likely to be location-specific, they still impact the healthcare available and received by trans persons and therefore influence their experiences and perception of healthcare. Most participant experiences related to healthcare systems were described negatively. Participants discussed a lack of policies and practices for trans healthcare, and criticised the waiting times (such as the length of the Real Life Experience) to access desired treatments (Bauer et al., 2009; Linander et al., 2017; Roller et al., 2015; Taylor, 2013; Vermeir et al., 2018). This reinforced participants’ feeling of vulnerability and dependence on HCPs (Linander et al., 2017). Participants also discussed experiencing complexities with medical, financial, and legal documentation related to name and gender, and the limitation of subsidised medical treatments to particular indications, diagnoses, or population groups (Bauer et al., 2009; Costa et al., 2018; Dewey, 2008; Hinrichs et al., 2018; Lindroth, 2016; Pitts et al., 2009; Ross \& Bell, 2017; Shires \& Jaffee, 2015; von Vogelsang et al., 2016). This was also expressed when participants described their experiences of accessing sex-specific or sex-segregated medical services (Bauer et al., 2009; Lindroth, 2016; Pitts et al., 2009; Vermeir et al., 2018). 


\section{Subtheme and Peripheral Themes}

Perceived underlying cisnormativity and transphobia. The above four themes were attributed by participants to be functions of the subtheme of underlying cisnormativity and transphobia (Bauer et al., 2009; Dewey, 2008; Hudson, 2018; Kosenko et al., 2013; Poteat et al., 2013; Taylor, 2013; Vermeir et al., 2018; Westerbotn et al., 2017). Transphobia, stigma, and discrimination towards trans individuals were perceived in relationship dynamics and through HCP communication. This was thought to reinforce the societal value of cisnormativity, or the “dominant gender binary ideology” (Taylor, 2013, p. 102), which participants perceived through the structure of healthcare systems, and in the lack of HCP education about the trans population. The participants' negative experiences reinforced this perceived underlying cisnormativity and transphobia, and influenced the choices they made about their healthcare and the recommendations they provided.

Choices. The above themes and subtheme influenced how trans patients felt about and perceived their healthcare experience, thus resulting in the first peripheral theme of decision making towards either self-empowerment or tolerance of the perceived underlying transphobia and cisnormativity depending on their other available healthcare options.

Tolerance involved participants lowering their standards for trans healthcare and making sacrifices to obtain their desired care (Bauer et al., 2009; Dewey, 2008; Poteat et al., 2013; Roller et al., 2015; Taylor, 2013). This included expecting lesser treatment, altering their expectations of what they perceive as competent care, and reducing their demands to better navigate the HCPs' lack of trans knowledge (Dewey, 2008; Poteat et al., 2013; Westerbotn et al., 2017). Poteat et al. (2013) provided an example where the trans patient may request the HCP to refill an existing prescription unchanged instead of asking for a dosage review or the perceived standard medical monitoring. Participants also reported anticipating discrimination when meeting HCPs and tolerating difficulties such as distance 
and waiting times to receive the perceived competent care (Bauer et al., 2009; Poteat et al., 2013; Roller et al., 2015; Taylor, 2013; Westerbotn et al., 2017). Tolerance also included enduring HCP questions and/or educating their HCP to maintain an established relationship with an unknowledgeable provider and avoid having to begin the healthcare journey anew (Hudson, 2018; Roller et al., 2015; Vermeir et al., 2018). Participants also reported being selective with the information they disclosed to their HCP in order to improve their likelihood of receiving desired treatments, concealing their trans identity when accessing non-trans healthcare, and/or delaying or avoiding sometimes necessary medical services to avoid perceived or actual discrimination (Bauer et al., 2009; Costa et al., 2018; Dewey, 2008; Lindroth, 2016; Taylor, 2013; Westerbotn et al., 2017).

Self-empowerment as a response to perceived transphobia and cisnormativity was demonstrated by self-advocacy, with participants adopting the responsibility for their own trans-related care (Dewey, 2008; Linander et al., 2017; Taylor, 2013). This involved the previously discussed self-education, and networking with others in the trans community to obtain and share knowledge, experiences and resources required for trans healthcare (Dewey, 2008; Poteat et al., 2013; Roller et al., 2015; Taylor, 2013; Vermeir et al., 2018). One participant in Poteat et al.’s 2013 study demanded better care from their HCP stating “these are unacceptable standards of care ... you need to prepare yourself for different types of people walking into your office” (p. 26), while others terminated their relationships with unsatisfactory HCPs, moving on to seek trans-competent providers (Poteat et al., 2013; Roller et al., 2015). Some participants also self-treated with hormones obtained online instead of relying on the healthcare system (Dewey, 2008; Linander et al., 2017).

Participant recommendations. Participants provided recommendations addressing the four main themes to improve their interactions with HCPs and the healthcare system (Bauer et al., 2009; Guss et al., 2017; Pitts et al., 2009; Ross \& Bell, 2017; Taylor, 2013; 
Vermeir et al., 2018); this formed the second and final peripheral theme. Some suggestions were for the HCP to confidentially ask for preferred pronouns and name at every visit, (Bauer et al., 2009; Guss et al., 2017) and to increase education for HCPs about trans health to improve awareness about gender diversity and the potential for patients to be trans (Bauer et al., 2009; Guss et al., 2017; Pitts et al., 2009; Taylor, 2013; Vermeir et al., 2018). Participants in Taylor (2013) suggested a centralised information database as a clinical resource for trans health. Environmental friendliness in healthcare spaces such as the display of posters or other visible symbols of trans-friendliness was a recommendation by participants in the 2009 Bauer et al. study, in Guss et al.’s 2017 study, and in Vermeir et al.’s 2018 study. In Ross \& Bell (2017), this extended to online websites. Regarding healthcare systems, participants from the 2009 Bauer et al. study suggested elimination of sex-segregation where possible or inclusion of trans-specific spaces in sex-segregated services, protocols for healthcare that do not assume cissexuality, removal of sex designations from medical documentation unless necessary, and resources for referral to trans-friendly providers. Participants in Ross \& Bell (2017) suggested adding the option of transgender on medical intake forms. The simplest summary of these recommendations was given by participants in Pitts et al.’s 2009 study, which suggested “proper trans community consultation” (p. 489).

\section{Discussion}

This review synthesised the trans perspectives and experiences of general healthcare in 20 studies. The main findings of this review included four recurring themes of HCP knowledge, HCP communication, relationship dynamics, and healthcare systems. Underlying these four themes was the subtheme of perceived cisnormativity and transphobia, leading to the first peripheral theme of trans patients choosing to tolerate and/or resist these underlying notions through self-empowerment depending on their available options and the potential for 
perceived discrimination. Trans patients also provided recommendations on how to improve their healthcare which formed the second peripheral theme. These findings are similar to those of McCann \& Sharek’s 2016 review of 10 studies addressing trans experiences with mental health services. This review found four main themes describing challenges which existed regarding mental health service provision. These included: issues accessing appropriate and quality trans-tailored services free of discrimination and transphobia; practitioner responsiveness, sensitivity, and affirmation; family and partner support provision during the process of transition; and potential developments for mental health services in order to address the scarcity of services and dissatisfaction with existing services. Despite having a specific rather than general healthcare focus, there is significant overlap in the findings between this review and that of the current review. Similarly, Lerner \& Robles’ 2017 review focusing on perceived barriers and facilitators to healthcare utilisation for trans people in the US also reported similar findings of a lack of HCP knowledge regarding trans identity and health issues, previous negative experiences with the healthcare system and/or anticipation of these experiences, inability to finance healthcare services, and refusal of HCPs to provide services to trans people as barriers to healthcare utilisation. These findings align with the described avoidance behaviour regarding tolerance and self-empowerment as a response to perceived transphobia and cisnormativity. The findings of a literature summary focused on the predisposing, reinforcing, enabling factors of trans-positive clinical behaviour change also supported the findings of these reviews, and aligned with the recommendations of improved HCP education and clinical resources suggested by participants in the current review (MacKinnon, Tarasoff, \& Kia, 2016).

All studies included in the current review described perceived negative experiences, suggesting a high prevalence of negative experiences in trans healthcare. This is supported by the 75.3\% of respondents in Bauer et al.'s 2015 survey who reported at least one trans- 
specific negative experience. Interestingly, some trans participants attributed HCP awkwardness and/or insufficient information provision as deliberate acts of transphobia rather than attributing these behaviours to other factors. (Kosenko et al., 2013; von Vogelsang et al., 2016) As some trans patients anticipate discrimination in healthcare encounters, (Poteat et al., 2013) this may lower the threshold for perceiving a healthcare interaction as negative and potentially skew the trans patient's perspective of what may or may not be attributed to transphobia and/or cisnormativity. Trans patients may also enter the healthcare encounter with unrealistic expectations and/or preconceived ideas of the care they wish to receive. In some cases, it may not be unreasonable for some healthcare fields (e.g. family practice) to lack knowledge about transition-specific care (e.g. hormone regimens). However, this may not meet the patient's expectations thus resulting in a perceived negative experience. Fortunately, some studies also described perceived positive experiences suggesting that not all healthcare encounters are bleak. Furthermore, the finding of trans patients educating themselves and their HCPs and choosing whether to remain with an established HCP or find another perceived to be more competent suggests empowerment and self-determination. In addition, the finding that partnerships improved healthcare relationships supports established literature about patient-centred care and collaboration, and the importance of this to patient satisfaction and improved patient outcomes (Gluyas, 2015; Delaney, 2018).

As multiple reviews and literature summaries provide similar findings from differing foci, there is little doubt about the perceived reality of these experiences for trans patients. These findings clearly indicate the need for greater consideration of the healthcare delivery to trans patients at both the healthcare provider and systems levels. This should take into account the implications of perceived cisnormativity and transphobia on trans patients' healthcare experiences, and the appropriateness and feasibility of participant recommendations. The next step is for HCPs at the user-interface and policy makers at the 
systems level to not only acknowledge the voiced experiences of these trans patients, but also consider their suggestions to improve provider knowledge, trans healthcare delivery, and the resultant health of trans people.

\section{Limitations}

A limitation of the studies was their geographical location. All studies had a Westerncountry focus, the majority being US-centric, and some studies addressed location-specific healthcare structures, so applicability may be limited to other locations and cultures. Additionally, many studies recruited participants from urban and/or metropolitan areas. Therefore, the needs and demands for healthcare delivery may not be applicable to rural locations, and it is possible some issues may not have been uncovered. Many of the qualitative studies did not address researcher reflexivity upon their data collection, analysis and results, and therefore it is unknown whether any bias was accounted for at any of these stages. This may have skewed the interpretation and synthesis of findings within the current review. Also, half of the mixed methods studies did not address their mixed methods design and their quantitative aspects were not discussed. The focus on negative healthcare experiences in most of the reviewed studies (and in some studies, the emotionally charged language) may also present a skewed perspective of an outspoken or wronged sample of participants and may not present a true reflection of positive healthcare experiences or the amount at which these may occur.

Regarding the review, limitations included its strict inclusion and exclusion criteria that could bias the selection of the studies included. For example, any trans experiences of healthcare that may have been included in studies also including lesbian, gay and bisexual non-trans participants would have been excluded. Additionally, other non-trans non-binary or gender non-conforming identities were excluded based on the criteria. In addition, this review 
excluded certain healthcare fields and social services which may have contained rich data related to the topic. This review also did not stratify data between male-identifying or femaleidentifying trans people, and did not account for social determinants such as income or race. As this review had a general healthcare focus, specific fields (e.g. psychiatry) may require further reviews or research to address field-specific needs or assess the depth of literature. A limitation affecting the synthesised results was the inclusion of Guss et al. (2017) which was still in progress. Therefore the preliminary findings which were included in analysis may be less rich than what the study will come to find once complete, and may limit the synthesis of what was found in this review and influence the findings of future reviews on similar topics. Lastly, this review was conducted predominantly by a single person under time and resource constraints. This impacted the study selection criteria and the depth of quality appraisal and analysis of the literature. There is also inherent reporting bias associated with a thematic analysis performed by one person.

\section{Conclusion}

Transgender people commonly report negative perceptions of their experiences in the research addressing their healthcare experiences. This emerged through the four main themes of healthcare provider knowledge, healthcare provider communication, relationship dynamics, and healthcare systems. Ultimately, these experiences reflected a subtheme of underlying notions of perceived cisnormativity and transphobia. Implications of this included trans patient self-education and self-empowerment, tolerance of these notions to avoid conflict in healthcare interactions and receive desired care, and/or the potential for poorer health due to unstable healthcare relationships. Recommendations from the trans patient to improve their healthcare experience were largely based in combating the perceived cisnormativity and transphobia. The findings of this review suggest the need for greater 
education of HCPs and healthcare staff about the trans population, perhaps through changes to medical curricula and/or mandatory staff training about patient-centred care and ways in which to sensitively interact with patients to minimise the interpersonal barriers related to accessing care. While this review provides a synthesis of themes surrounding trans patient experiences with general healthcare, further research is needed to examine the issues surrounding healthcare delivery for trans patients in particular geographic locations, affecting particular demographics, and in other specific areas of healthcare or with particular healthcare personnel. Additionally, an examination of HCP experiences with trans patients may also be beneficial to synthesise a detailed picture of trans healthcare encounters. Findings of future studies would facilitate targeted and holistic implementation of interventions and recommendations to improve healthcare delivery for trans people.

\section{Funding}

This review was not funded.

\section{Conflict of Interest}

The authors declare that they have no conflict of interest. 


\section{Indication of figures and tables}

\section{Tables}

- Table 1: Characteristics of included studies

- Table 2: Descriptive quality appraisal of included studies

- Table 3: Quality appraisal criteria adapted from Pluye et al., 2009.

- Table 4: Quality appraisal of included studies adapted for improved visual comprehension.

\section{Figures}

- Figure 1: Flow chart of the searching and screening process.

- Figure 2: Relationship between identified themes, subtheme, and peripheral themes. 


\section{References}

American Psychological Association. (2015). Key Terms and Concepts in Understanding Gender Diversity and Sexual Orientation Among Students. In American Psychological Association (Ed.), (Vol. 5): American Psychological Association.

Bauer, G. R., Hammond, R., Travers, R., Kaay, M., Hohenadel, K. M., \& Boyce, M. (2009). "I don't think this is theoretical; this is our lives": how erasure impacts health care for transgender people. Journal of the Association of Nurses in AIDS Care, 20(5), 348361. doi:10.1016/j.jana.2009.07.004

Bauer, G. R., Zong, X. C., Scheim, A. I., Hammond, R., \& Thind, A. (2015). Factors Impacting Transgender Patients' Discomfort with Their Family Physicians: A Respondent-Driven Sampling Survey. PLoS ONE, 10(12), 16. doi:10.1371/journal.pone.0145046

Costa, A. B., da Rosa, H. T., Pase, P. F., Fontanari, A. M. V., Catelan, R. F., Mueller. A., ... Koller, S. H. (2018). Healthcare Needs of and Access Barriers for Brazilian Transgender and Gender Diverse People. Journal of Immigrant and Minority Health, 20(1), 115-123. doi: 10.1007/s10903-016-0527-7

de Haan, G., Santos, G. M., Arayasirikul, S., \& Raymond H. F. (2015). Non-Prescribed Hormone Use and Barriers to Care for Transgender Women in San Francisco. LGBT Health, 2(4), 313-323. doi: 10.1089/lgbt.2014.0128

Delaney, L. J. (2017). Patient-centred care as an approach to improving health care in Australia. Collegian, 25(1), 119-123. doi:10.1016/j.colegn.2017.02.005

Dewey, J. M. (2008). Knowledge legitimacy: how trans-patient behavior supports and challenges current medical knowledge. Qualitative Health Research, 18(10), 13451355. doi:10.1177/1049732308324247

Ellis, S., Bailey, L., \& McNeil, J. (2015). Trans peoples' experiences of mental health and gender identity services: a UK study. Journal of Gay \& Lesbian Mental Health, 19(1), 4-20. doi:10.1080/19359705.2014.960990

GLAAD. Transgender FAQ. Retrieved from https://www.glaad.org/transgender/transfaq Gluyas, H. (2015). Patient-centred care: improving healthcare outcomes. Nursing Standard, 30(4), 50-57; quiz 59. doi:10.7748/ns.30.4.50.e10186

Guss, C. E., Woolverton, A., Borus, J., Reisner, S. L., Austin, S. B., \& Katz-Wise, S. L. (2017). “Just Step Up:” A Qualitative Study of Transgender Adolescents’ Experiences 
in Primary Care. Journal of Adolescent Health, 60, S28-S29.

doi:10.1016/j.jadohealth.2016.10.074

Hinrichs, A., Link, C., Seaquist, L., Ehlinger, P., Aldrin, S., \& Pratt, R. (2018). Transgender and Gender Nonconforming Patient Experiences at a Family Medicine Clinic. Academic Medicine, 93(1), 76-81. doi:10.1097/ACM.0000000000001837

Hudson, K. D. (2018). (Un)doing Transmisogynist Stigma in Health Care Settings: Experiences of Ten Transgender Women of Color. Journal of Progressive Human Services, 1-19. doi:10.1080/10428232.2017.1412768

Kosenko, K., Rintamaki, L., Raney, S., \& Maness, K. (2013). Transgender patient perceptions of stigma in health care contexts. Medical Care, 51(9), 819-822. doi:10.1097/MLR.0b013e31829fa90d

Lerner, J. E., \& Robles, G. (2017). Perceived Barriers and Facilitators to Health Care Utilization in the United States for Transgender People: A Review of Recent Literature. Journal of Health Care for the Poor and Underserved, 28(1), 127-152.

Linander, I., Alm, E., Hammarström, A., \& Harryson, L. (2017). Negotiating the (bio)medical gaze-Experiences of trans-specific healthcare in Sweden. Social Science \& Medicine, 174, 9-16. doi:10.1016/j.socscimed.2016.11.030

Lindroth, M. (2016). 'Competent persons who can treat you with competence, as simple as that'-An interview study with transgender people on their experiences of meeting health care professionals. Journal of Clinical Nursing, 25(23-24), 3511-3521. doi:10.1111/jocn.13384

MacKinnon, K. R., Tarasoff, L. A., \& Kia, H. (2016). Predisposing, reinforcing, and enabling factors of trans-positive clinical behavior change: A summary of the literature. International Journal of Transgenderism, 17(2), 83-92. doi:10.1080/15532739.2016.1179156

McCann, E., \& Sharek, D. (2016). Mental Health Needs of People Who Identify as Transgender: A Review of the Literature. Archives of Psychiatric Nursing, 30(2), 280285. doi:10.1016/j.apnu.2015.07.003

McNeil, J., Bailey, L., Ellis, S., Morton, J., \& Regan, M. (2012). Trans Mental Health Study 2012. Retrieved from Edinburgh, United Kingdom: https://www.gires.org.uk/assets/Medpro-Assets/trans_mh_study.pdf 
Moher D, Liberati A, Tetzlaff J, Altman D.G., The PRISMA Group (2009). Preferred Reporting Items for Systematic Reviews and MetaAnalyses: The PRISMA Statement. PLoS Med, 6(7), e1000097. doi:10.1371/journal.pmed1000097

Nobelius, A. (2004). What is the difference between sex and gender? Retrieved from http://www.med.monash.edu.au/gendermed/sexandgender.html

Pitts, M. K., Couch, M., Mulcare, H., Croy, S., \& Mitchell, A. (2009). Transgender People in Australia and New Zealand: Health, Well-being and Access to Health Services. Feminism \& Psychology, 19(4), 475-495. doi:10.1177/0959353509342771

Pluye, P., Gagnon, M. P., Griffiths, F., \& Johnson-Lafleur, J. (2009). A scoring system for appraising mixed methods research, and concomitantly appraising qualitative, quantitative and mixed methods primary studies in Mixed Studies Reviews. International Journal of Nursing Studies, 46(4), 529-546. doi:10.1016/j.ijnurstu.2009.01.009

Poteat, T., German, D., \& Kerrigan, D. (2013). Managing uncertainty: a grounded theory of stigma in transgender health care encounters. Social Science \& Medicine, 84, 22-29. doi:10.1016/j.socscimed.2013.02.019

Riggs, D. W., Coleman, K., \& Due, C. (2014). Healthcare experiences of gender diverse Australians: a mixed-methods, self-report survey. BMC Public Health, 14, 230. doi:10.1186/1471-2458-14-230

Roller, C. G., Sedlak, C., \& Draucker, C. B. (2015). Navigating the System: How Transgender Individuals Engage in Health Care Services. Journal of Nursing Scholarship, 47(5), 417-424. doi:10.1111/jnu.12160

Ross, K. A., \& Castle Bell, G. (2017). A Culture-Centered Approach to Improving Healthy Trans-Patient-Practitioner Communication: Recommendations for Practitioners Communicating with Trans Individuals. Health Communication, 32(6), 730-740. doi:10.1080/10410236.2016.1172286

Rosenstreich, G. (2013). LGBTI People Mental Health and Suicide. Retrieved from Sydney, Australia: https://www.beyondblue.org.au/docs/default-source/default-documentlibrary/bw0258-lgbti-mental-health-and-suicide-2013-2nd-edition.pdf?sfvrsn=2

Rotondi, N. K., Bauer, G. R., Scanlon, K., Kaay, M., Travers, R., \& Travers, A. (2013). Nonprescribed Hormone Use and Self-Performed Surgeries: “Do-It-Yourself” Transitions in Transgender Communities in Ontario, Canada. American Journal of Public Health, 103(10), 1830-1836. doi: 10.2105/AJPH.2013.301348 
Shires, D. A., \& Jaffee, K. (2015). Factors associated with health care discrimination experiences among a national sample of female-to-male transgender individuals. Health \& Social Work, 40(2), 134-141.

Sinclair, L., \& Leach, R. (2017). Exploring thoughts of suicide. BMJ, 356. doi:10.1136/bmj.j1128

Taylor, E. T. (2013). Transmen's health care experiences: Ethical social work practice beyond the binary. Journal of Gay \& Lesbian Social Services: The Quarterly Journal of Community \& Clinical Practice, 25(1), 102-120. doi:10.1080/10538720.2013.750575

Vermeir, E., Jackson, L. A., \& Marshall, E. G. (2018). Barriers to primary and emergency healthcare for trans adults. Culture, Health and Sexuality, 20(2), 232-246. doi:10.1080/13691058.2017.1338757

von Vogelsang, A. C., Milton, C., Ericsson, I., \& Strömberg, L. (2016). 'Wouldn’t it be easier if you continued to be a guy?'-A qualitative interview study of transsexual persons' experiences of encounters with healthcare professionals. Journal of Clinical Nursing, 25(23-24), 3577-3588. doi:10.1111/jocn.13271

White Hughto, J., Reisner, S., \& Pachankis, J. (2015). Transgender stigma and health: a critical review of stigma determinants, mechanisms, and interventions. Social Science \& Medicine, 147, 222-231. doi:10.1016/j.socscimed.2015.11.010.

Westerbotn, M., Blomberg, T., Renström, E., Saffo, N., Schmidt, L., Jansson, B., \& Aanesen, A. (2017). Transgender people in Swedish healthcare: The experience of being met with ignorance. Nordic Journal of Nursing Research, 37(4), 194-200. doi:10.1177/2057158517695712 
Table 1: Characteristics of included studies

\begin{tabular}{|c|c|c|c|c|}
\hline $\begin{array}{l}\text { Citation } \\
\text { (Country) }\end{array}$ & Sample \& Age & $\begin{array}{l}\text { Health Care } \\
\text { Focus }\end{array}$ & Method & Key Findings \\
\hline $\begin{array}{l}\text { Bauer et al., } \\
2015 \text { (Canada) }\end{array}$ & $\begin{array}{l}433 \text { transgender } \\
\text { people aged } 16 \\
\text { years \& older }\end{array}$ & $\begin{array}{l}\text { Family } \\
\text { physicians }\end{array}$ & $\begin{array}{l}\text { Quantitative - cross- } \\
\text { sectional survey }\end{array}$ & $\begin{array}{l}50 \% \text { experience discomfort discussing trans health issues - } \\
\text { increased with previous negative experience, decreased with } \\
\text { greater perceived physician knowledge about trans issues. } \\
75.3 \% \text { had at least } 1 \text { trans-specific negative experience. } \\
53.6 \% \text { told HCP did not know enough about trans-related } \\
\text { care to provide it. }\end{array}$ \\
\hline $\begin{array}{l}\text { Costa et al., } \\
2018 \text { (Brazil) }\end{array}$ & $\begin{array}{l}626 \text { TGD people } \\
\text { aged } 18-61 \text { years }\end{array}$ & $\begin{array}{l}\text { Trans-specific } \\
\text { services }\end{array}$ & $\begin{array}{l}\text { Quantitative - } \\
\text { hospital \& web- } \\
\text { based cross-sectional } \\
\text { survey }\end{array}$ & $\begin{array}{l}\text { Healthcare discrimination experiences significantly affected } \\
\text { the frequency of healthcare avoidance }(\mathrm{p}<0.001) \text {. } \\
\text { Approximately } 60 \% \text { felt uncomfortable discussing their } \\
\text { TGD health needs with the HCPs they accessed. Over } 60 \% \\
\text { stated they had to educate a HCP about their needs as a TGD } \\
\text { person. Approximately } 40 \% \text { avoided needed healthcare due } \\
\text { to being TGD. Around } 60 \% \text { had been discriminated against } \\
\text { in a healthcare context; the HCP not using the person's } \\
\text { preferred name was the most common situation. }\end{array}$ \\
\hline $\begin{array}{l}\text { Dewey, } 2008 \\
\text { (US) }\end{array}$ & $\begin{array}{l}22 \text { transgender } \\
\text { people. Mean age: } \\
48 \text { years }\end{array}$ & Not specified & $\begin{array}{l}\text { Qualitative - in-depth } \\
\text { interviews + } \\
\text { participant } \\
\text { observation at social } \\
\text { \& group functions }\end{array}$ & $\begin{array}{l}\text { Medical experiences affect trans patients' perception of their } \\
\text { medical treatment \& choice to alter behaviour in future } \\
\text { encounters. Experiences surround treatment by the HCP, } \\
\text { trans patients' approaches to HCP, \& understanding of } \\
\text { medical knowledge by both parties. }\end{array}$ \\
\hline
\end{tabular}


Guss et al., $\quad 7$ transgender $\quad$ Primary care

2017 (US) adolescents aged

between $13-21$

years (total of 20

to be recruited)

Hinrichs et al., $\quad 23$ TGNC adults in 2018 (US) focus groups, 22 in survey

Hudson, 2018

10 transwomen of
colour

Kosenko et al., 2013 (US)
Family medicine

LGBT-specific \& general healthcare
Qualitative interviews
Mixed - focus

groups + survey

Mixed (ongoing) demographic questionnaire + semistructured interview
Most participants had positive experiences with HCP \& found clinician accepting of gender identity. 6 main themes:

(a) Pronouns \& names; (b) Privacy; (c) Physical spaces; (d) Conversations with providers; (e) Provider knowledge; (f) Medical staff roles.

4 main themes: (a) negative healthcare experiences (misgendering, wrong name, assuming sex work or trauma, invalidation, stigma or rejection, systems); (b) need for sensitive \& inclusive primary care (holistic); (c) defining TGNC-sensitive care (acceptance, treat as a whole person, partnership); (d) challenges of mainstreaming TGNCcompetent care into primary care settings (consistent HCP competence, TGNC as educator).

LGBT: mostly positive - ‘whole person' approach, LGBT HCP, identity-conscious services (knowledge, acceptance), respectful \& comfortable subjective environment. Negative perceived less appropriate for health issues unrelated to sex or gender, sexual minority providers may not understand gender minority needs.

General: mostly negative - microaggressions (misgendering, behaviour, ignored), overemphasised LGBT identity \& mistrust, patient as educator (knowledge, denial of care), HCPs’ perceived personal biases. 1 positive experience treated as 'normal'.

71\% had at least 1 instance of mistreatment in healthcare. 6 themes of mistreatment: (a) Gender insensitivity; (b)

questionnaire (qualitative analysis)
Displays of discomfort; (c) Denied services; (d) Substandard care; (e) Verbal abuse; (f) Forced care. Attributed to 
Linander et al., 14 transgender 2017 (Sweden) people aged 23 69 years

Lindroth, 201620 transgender \& (Sweden) non-binary people aged $18-74$ years

Pitts et al., 2009 (Australia \& NZ)

\section{3 transgender} people aged 60 years \& below

\section{Trans-specific} health care

Sexual health

care

Other areas

(endocrinology, surgery, primary healthcare,

gynaecology \& more)

Not specified

Mixed - online survey (quantitative \& qualitative analysis of responses) transphobia \& stigma.

Qualitative interviews

Qualitative interviews

Trans-specific healthcare difficult to navigate due to: waiting times, lack of support, provider ignorance, and relationships of dependency between healthcare users \& providers.

Trans people perceive experiences of disrespect from HCPs. 3 themes: (a) Estrangement; (b) Expectations; (c) Eviction.
Poteat et al., 2013 (US)
55 transgender people aged $21-$ 66 years

\section{Not specified}

Qualitative - in-depth interviews
Best experiences: felt accepted \& supported; showing empathy, understanding, compassion, professionalism (nonjudgemental, respectful, appropriate use of pronouns/names, documentation), sensitivity, knowledgeable HCPs.

Worst experiences: hostility, discomfort, contempt, refused treatment, ridiculed, disgust.

Sex-specific medical clinics: difficult experience, avoidance, worse if HCP not respectful.

Some never spoke to HCP about trans-related issues, often reluctant.

Trans people anticipate providers do not know how to meet needs. Key themes: uncertainty \& ambivalence which upsets the normal balance of power (reinforced authority of medical professionals). 
Riggs et al., 2014

(Australia) 2015 (US)

Ross \& Bell, 2017 (US) 2015 (US) aged $25-44$ years people (defined as a mismatch between birth sex/genitalia \& gender identity)

\section{5 transgender} people aged 21 64 years

12 transgender people aged 18 years or older
Shires \& Jaffee, 1,711 transmen
188 gender diverse

\section{Outpatient} Hospital

Any aspects of participants' healthcare experiences

Unspecified physicians
Mixed - online survey (quantitative and qualitative analysis of responses)

Qualitative interviews

Qualitative - semistructured interviews

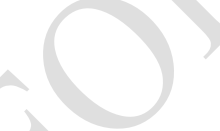
sectional survey data

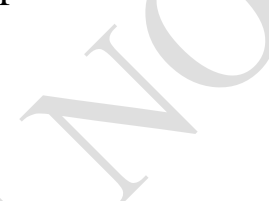

Taylor, 2013

3 transmen aged 21 "All processes, (Canada)

Qualitative - semistructured interviews

\section{practices, and}

sites in which

health care is

offered or

performed"
Quantitative - cross-

Positive experiences: professional, helpful, knowledgeable, caring/humane, comfort with HCP, treated with respect, HCP willing to work with trans person.

Negative experiences - gatekeeping, offensive questions, perceived invasive/abuse physical health care, need to educate HCP, discrimination, confidentiality breaches.

Central phenomenon of how trans people engage in health care: navigating the system. Involves 4 sub-processes: (a) Needing to move forward; (b) Doing due diligence; (c) Finding loopholes; (d) Making it work.

2 main themes: (a) advice for HCPs - communication behaviour modifications (e.g. acknowledge trans status only if relevant, use preferred pronouns, as only health- or treatment-relevant questions); (b) office procedures - adjust intake forms, modify online material to indicate transfriendly or -focused expertise.

$41.8 \%$ report verbal harassment, physical assault, denial of equal treatment in a doctor's office or hospital.

Some factors associated with increased or decreased reporting of healthcare discrimination experiences (e.g. income, race, sexual orientation, etc.).

Transmen perform extra work to compensate for a lack of provider competence in trans health care. Extra work in the areas of knowledge gathering, quality of helping relationships, access to health interventions. Dominant gender binary ideology shapes healthcare experiences. 
Vermeir et al., 8 transgender 2018 (Canada) adults

von Vogelsang
et al., 2016

(Sweden)

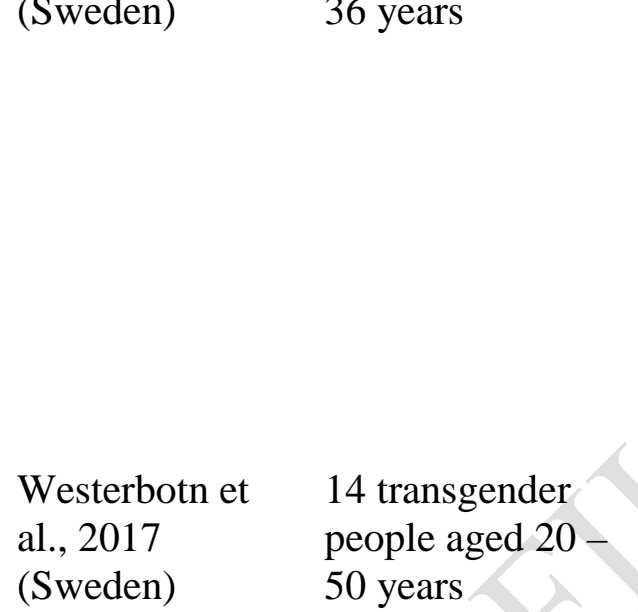

Primary \&

emergency care
Qualitative - semistructured interviews
Primary data explored both barriers and facilitators to care, however authors focused on key barriers to care leading to perceived discrimination, invisibility \& disrespect. (a) Interpersonal barriers - HCP knowledge (lacking, inappropriate resources, patient as educator), HCP \& staff sensitivity \& appropriateness (attitudes, wrong name/pronoun, being outed, terminology, inappropriate or irrelevant questions or care). (b) Physical environment barriers - rural less tolerant, privacy, intake forms, lack of gender-neutral amenities, trans-related information or transfriendly indicators. (c) Social environmental barriers -

'fallacious social discourses', waiting \& accessibility issues, lack of trans-health in HCP curriculum.
Sex Qualitative -

reassignment process semi-structured

interviews
3 categories (15 subcategories) to describe transsexual persons' experiences of encounters with HCPs during sex reassignment process. (a) Good encounter (Professionalism, Integrity \& respect, Responsiveness, Trust \& confidence, Trans person's own part in encounter); (b) HCP attitudes \& caregiving (Level of knowledge, How they speak to you \& about you; Exploiting position of power; Withholding information; Gender stereotypes); (c) Perceptions of vulnerability (Condescending view of oneself; Dependence; Can't choose not being trans; Not being taken seriously; External high demands).

Healthcare not related to gender

Qualitative - semistructured interviews reassignment with healthcare \& rarely or never encountering direct discrimination by HCPs. Most thought ignorance \& lack of knowledge affected HCP attitude. Subthemes: (a)
Lack of HCP knowledge, but mostly positive experiences 
Experience of treatment by HCPs: treated like everyone else (although expect ignorance, discrimination \& malpractice); appreciate a lack of unnecessary focus on trans-identity, but also no avoidance of trans-identity; appreciate use of correct pronouns \& name; acknowledge ignorance \& ask questions if knowledge gap is relevant to adequate care. (b)

Expectations of meeting HCPs: feeling anxious beforehand; consequences of expectations. (c) Consequences of meeting HCPs: avoid care; feeling exposed; questioned by HCPs.

Notes: US = United States; NZ = New Zealand; HCP = healthcare provider; TGD = transgender and gender diverse; TGNC = transgender and gender nonconforming; LGBT = lesbian, gay, bisexual, and transgender. 
Table 2: Descriptive quality appraisal of included studies

\section{Study}

Bauer et al., 2009

Dewey, 2008

Hudson, 2018

Linander et al., 2017

Lindroth, 2016

Poteat et al., 2013

Roller et al., 2015

Ross \& Bell, 2017

\section{Rigour}

Research aims clear \& qualitative. Methodology, design appropriate. Context described. Participants described and recruitment strategy appropriate. Data collection and analysis appropriate and described. Minimal mention of reflexivity.

Research aims somewhat clear, but qualitative methodology and design seems appropriate; design minimally described. Context described. Recruitment strategy not described but participants described. Data collection not discussed and analysis minimally discussed. Reflexivity not discussed.

Clear qualitative aim. Appropriate methodology and design. Context clearly described. Participants clearly described, and sampling justified. Qualitative data collection and analysis well-described. Researcher reflexivity well-discussed.

$$
20
$$

Qualitative aim clear. Qualitative approach/design/method appropriate (semi-structured interviews). Context clearly described (Sweden). Participants described and recruitment and sampling discussed and justified. Thorough description of qualitative data collection and analysis. Reflexivity discussed.

Qualitative aim clearly stated. Qualitative approach/design/method appropriate (interviews). Context described. Participants described and sampling justified. Qualitative data collection and analysis described (grounded theory). Researchers' reflexivity not discussed.

Clear qualitative aim. Appropriate qualitative design and method. Context described. Participants described and sampling justified. Qualitative data collection and analysis described. Reflexivity discussed.

Clear qualitative objective. Qualitative approach/design/method appropriate. Context described. Participants described and sampling justified. Qualitative data collection and analysis described. Researchers’ reflexivity briefly discussed and considered.

Thorough and clear qualitative research questions, with an appropriate design and methodology. Context described. Clear description of participants, and sampling well-justified. Thorough description of qualitative data collection and analysis. Reflexivity thoroughly addressed. 
Taylor, 2013

Qualitative objective. Appropriate qualitative approach/design/method. Context described. Participants described and sampling justified. Qualitative data collection and analysis described. Reflexivity not discussed.

Vermeir et al., 2018 Clear qualitative objective with an appropriate methodology. Context clearly described. Participant description and sampling clearly stated. Data collection and analysis well-described. Reflexivity not discussed.

von Vogelsang et al., Clear qualitative aim. Appropriate qualitative approach/design/method. Context described. Participants described and 2016 sampling justified. Qualitative data collection and analysis clearly described. Researchers' reflexivity not discussed.

Westerbotn et al., Clear qualitative aim with an appropriate design and methodology. Context clearly described. Clear description of 2017 participants, and sampling justified. Qualitative data collection and analysis well-described. Researchers' reflexivity not discussed.

\section{Quantitative observational}

Bauer et al., 2015 Clear background/rationale and objectives with hypotheses given. Study design, participant eligibility and recruitment clear. Variables clearly defined with description of handling. Bias addressed. Statistical methods described. Limitations addressed.

Costa et al., 2018

Clear statement of background/rationale and research objectives. Study design, participant eligibility and recruitment clear with appropriate sampling. Variables defined with description of statistical analyses applied. Justification of numbers presented, however unclear or inaccurate in text description of results (e.g. an $n$ value may not equate to the reported percentage given the declared total n value). Total sample divided into ‘trans women', 'trans men' and 'gender diverse people’ groups, otherwise minimal apparent controlling for confounders. However, limitations thoroughly addressed.

Shires \& Jaffee,

Clear background/rationale and objectives. Sample and sampling discussed and appropriate. Measurements justified, 2015 statistical analysis described. Statistical methods described. Limitations addressed. Relationship between many variables addressed. 


\section{Mixed methods}

Guss et al., 2017

Hinrichs et al., 2018

Kosenko et al., 2013 Qualitative objective clearly stated. Method somewhat appropriate (online survey with demographic questions, initial closed-ended screening question followed by open-ended questions). Context described. Participants described in results, sampling not discussed. Qualitative data collection and analysis clearly described. Reflexivity not discussed.

Quantitative sampling not discussed. Minimal discussion of quantitative measurements and analysis. Confounding variables not discussed.

Mixed methods design not discussed or justified. Combined quantitative data collection methods with quantitative and qualitative analysis. Qualitative and quantitative data and results integrated.

Pitts et al., 2009
Qualitative objective/question with aims clearly. Qualitative approach/design and method appropriate. Context described. Participants described with appropriate recruitment strategy. Described qualitative data collection and brief description of analysis. Reflexivity not addressed.

Quantitative observational demographic questionnaire - minimal discussion of sample, no justification of measurements, confounding variables not discussed.

Did not discuss or justify mixed methods design. Did not describe quantitative data analysis, but quantitative and qualitative methods were described. Quantitative and qualitative data were integrated in results (preliminary).

Q aim clearly stated. Methodology appropriate (demographic survey and focus group discussions). Conte clearly and thoroughly described. Participants described in results, justification of sampling discussed. Qualitative data collection and analysis clearly described. Reflexivity not discussed.

Quantitative demographic survey - minimal discussion of sample and sampling, minimal discussion of quantitative measurements and analysis (although software stated), confounding variables not controlled but limitations of sample clearly discussed.

No discussion or justification of mixed methods design. Minimal integration of qualitative and quantitative data collection-analysis techniques (short demographic survey before focus groups was the most procedural integration). Minimal discussion of quantitative results, and hardly integrated with qualitative data which made up the bulk of the results; however, some integration was made when discussing study limitations.
Study aim not clearly stated or qualitative. Qualitative aspects described, but approach predominantly quantitative (survey). Context described. Participants described and sampling justified. Qualitative data collection and analysis described. Reflexivity not discussed. 
Riggs et al., 2014

Quantitative sampling and sample appropriate. Measurements justified. Confounding variables not discussed. Mixed methods design justified (although not stated as such). Qualitative and quantitative data collection and analysis techniques combined. Qualitative and quantitative results integrated.

Study aims qualitative. Qualitative approach/design/method appropriate for mixed method study. Context described. Participants described and sampling justified. Qualitative data collection and analysis described. Reflexivity not discussed.

Quantitative sampling and sample appropriate. Measurements justified. Confounding variables accounted for and considered.

Mixed methods design justified. Qualitative and quantitative data collection and analysis techniques combined.

Qualitative and quantitative results integrated.

Notes: the criteria are described in Table 3. 
Table 3: Quality appraisal criteria adapted from Pluye et al., 2009.

\begin{tabular}{ll}
\hline $\begin{array}{l}\text { Qualitative } \\
\text { 1. Qualitative objective or } \\
\text { question. }\end{array}$ & $\begin{array}{l}\text { Poorly defined or unclear } \\
\text { qualitative objective or question. }\end{array}$ \\
$\begin{array}{l}\text { 2. Appropriate qualitative } \\
\text { approach or design or method. }\end{array}$ & $\begin{array}{l}\text { Approach or design or method is } \\
\text { not qualitative or is inappropriate } \\
\text { for the question or objective. }\end{array}$
\end{tabular}

3. Description of the context.

4. Description of participants and justification of sampling.

5. Description of qualitative data collection and analysis. 6. Discussion of researchers'
reflexivity.

Quantitative experimental

\section{Appropriate sequence}

generation and/or randomization.

\section{Context not described poorly} described, or unclear.

Both description of participants and justification of sampling weak, unclear, or not stated.

Qualitative data collection and analysis not described or very weakly described.

No or minimal discussion of researchers' reflexivity.

Inappropriate sequence generation and/or randomization.

\section{Satisfactory}

Partially defined qualitative objective or question.

Approach or design or method is somewhat appropriate for the question or objective.

Context somewhat described.

Description of participants and/or justification of sampling addressed, but lacking details; or description of participants welldescribed but sample justification not stated, or vice versa.

Description of qualitative data collection and analysis present, but lacking details.

Some discussion of researchers' reflexivity, but lacking.

Somewhat appropriate sequence generation and/or randomization.

\section{Good}

Thorough and clear qualitative objective or question.

Approach or design or method is clearly appropriate for the question or objective.

Context clearly described.

Both description of participants and justification of sampling welldescribed and clearly stated.

Qualitative data collection and analysis well-described.

Thorough discussion of researchers' reflexivity.

Clearly appropriate sequence generation and/or randomization. 
2. Allocation concealment and/or blinding.

3. Complete outcome data and/or low withdrawal/drop-out.

\section{Quantitative observational}

1. Appropriate sampling and sample.

2. Justification of measurements (validity and standards).

\section{Control of confounding variables.}

\section{Mixed methods}

1. Justification of the mixed methods design.

2. Combination of qualitative and quantitative data collectionanalysis techniques or procedures.

3. Integration of qualitative and quantitative data or results.
Allocation concealment and/or blinding not performed.

Outcome data incomplete and/or high withdrawal/drop-out.

Inappropriate sampling and sample.

No justification or poor justification of measurements (validity and standards).

Confounding variables not controlled or accounted for.

Mixed methods design not justified, very poorly justified, or inappropriate.

No combination of qualitative and quantitative data collection-

analysis techniques or procedures.

No or very weak integration of qualitative and quantitative data
Some allocation concealment and/or blinding evident or inappropriate.

Outcome data somewhat complete and/or fairly high withdrawal/drop-out.

Somewhat appropriate sampling and sample.

Measurements (validity and standards) somewhat justified.

Some control or accounting for confounding variables.

Some justification of the mixed methods design, or mixed methods design somewhat appropriate.

Some combination of qualitative and quantitative data collectionanalysis techniques or procedures.

Some integration of qualitative and quantitative data or results.
Allocation concealment and/or blinding performed appropriately.

Complete outcome data and/or low withdrawal/drop-out.

Appropriate sampling and sample.

Clear and thorough justification of measurements (validity and standards).

Thorough control of and account for confounding variables.

Clear justification of the mixed methods design, and mixed methods design clearly appropriate.

Well-combined qualitative and quantitative data collectionanalysis techniques or procedures.

Good integration of qualitative and quantitative data or results. 
or results.

Notes: the criteria used to assess mixed methods studies combine the qualitative criterion, quantitative experimental or observational criterion, as well as the mixed methods criterion. 
Table 4: Quality appraisal of included studies adapted for improved visual comprehension.

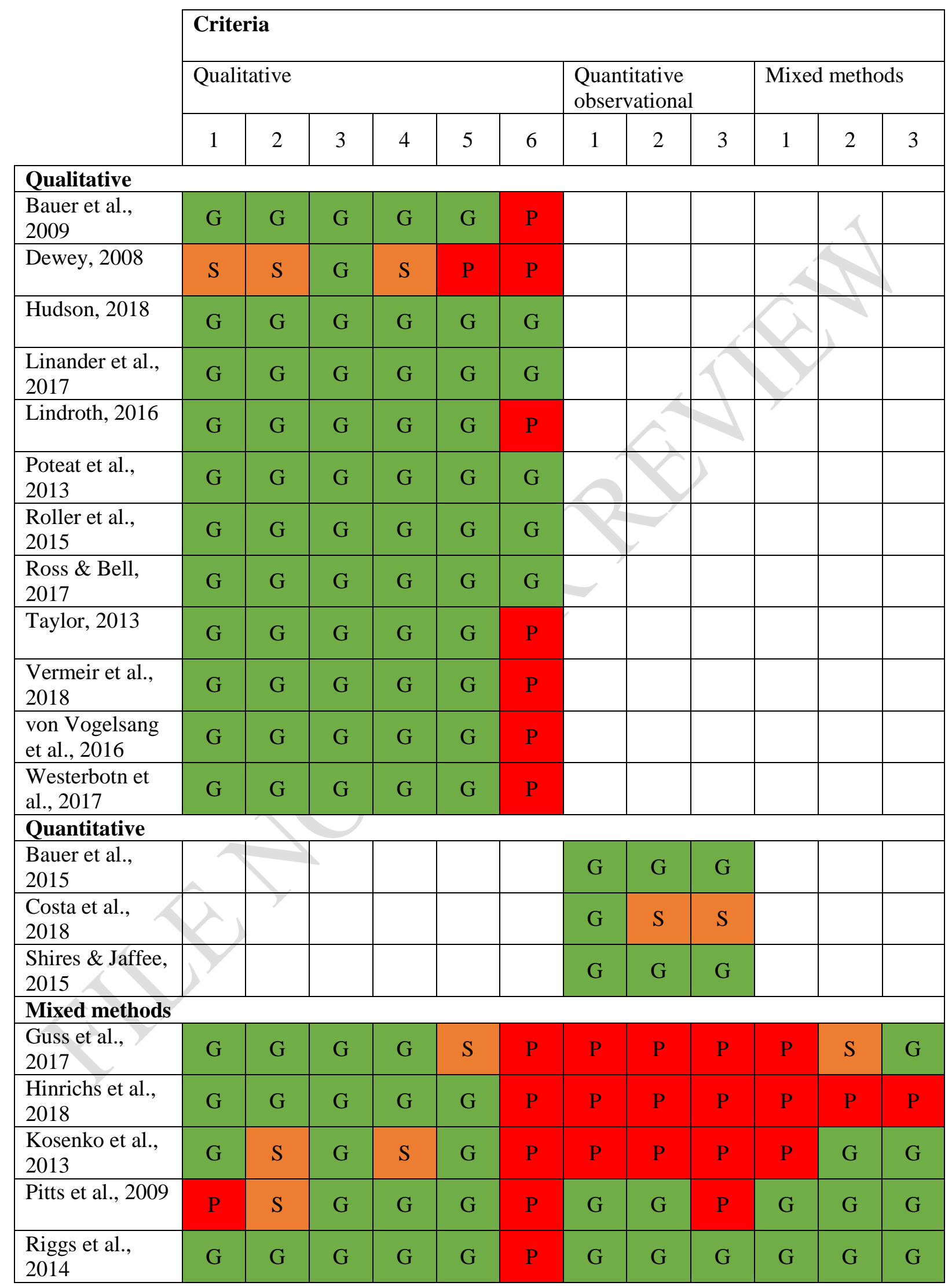

Notes: $\mathrm{P}=$ poor; $\mathrm{S}=$ satisfactory; $\mathrm{G}=$ good. Detailed appraisal criteria can be found in Table 3 

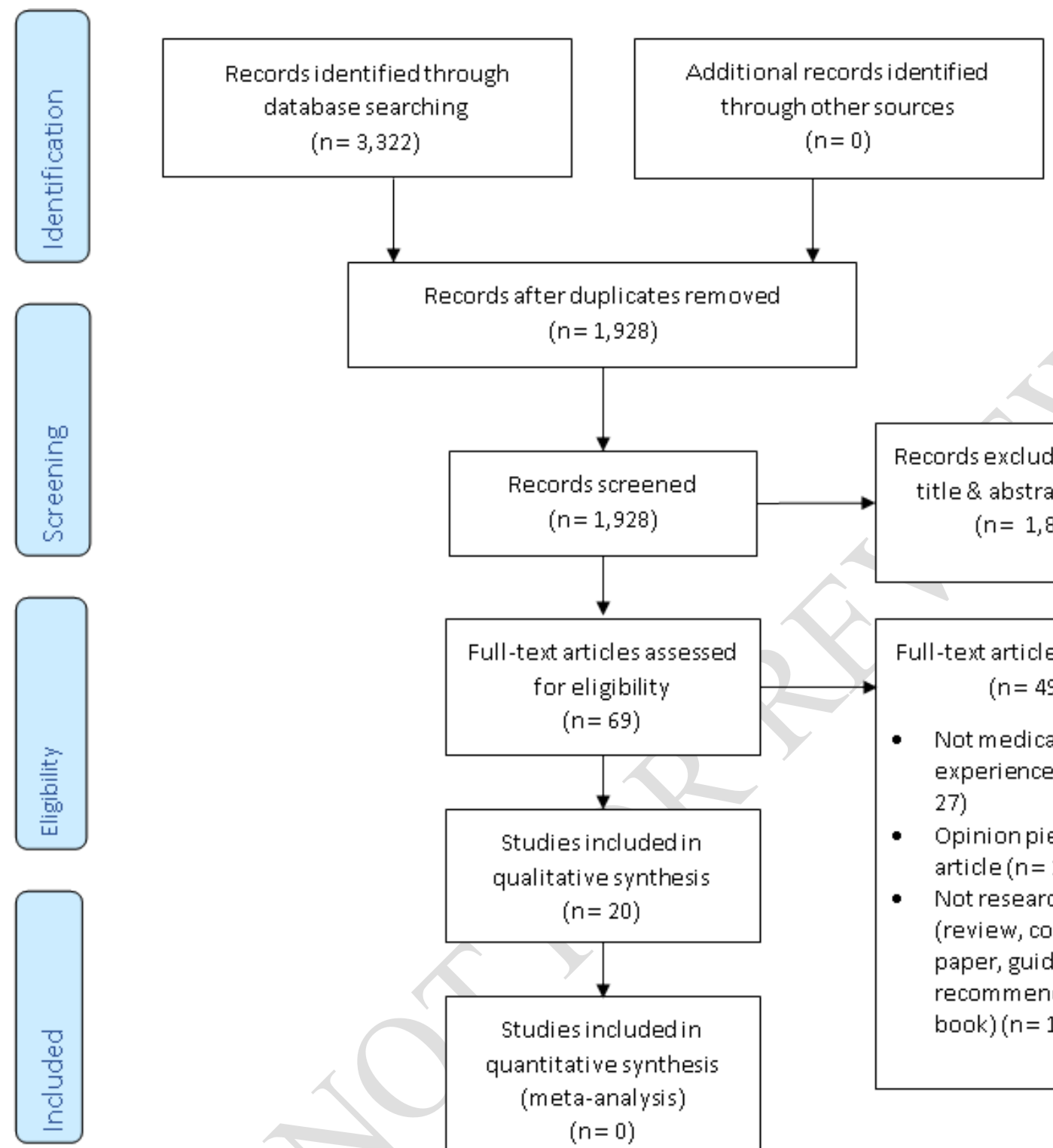

Records excluded through title \& abstract search $(n=1,859)$

Full-text articles assessed for eligibility $(n=69)$

Full-text articles excluded

$$
(n=49) \text { : }
$$

- Not medical experience focus $(n=$ 27)

Studies included in qualitative synthesis $(n=20)$

- Opinion piece, feature article $(n=10)$

- Not research article (review, conference paper, guideline, recommendation,

Studies included in quantitative synthesis book) $(n=12)$

(meta-analysis)

$$
(n=0)
$$

Notes: Figure adapted from Preferred Reporting Items for Systematic Reviews and MetaAnalyses: The PRISMA Statement (Moher, Liberati, Tetzlaff, Altman, The PRISMA Group, 2009). Appendix describes the search strategy and total results for each database. 
Figure 2: Relationship between identified themes, subtheme, and peripheral themes.

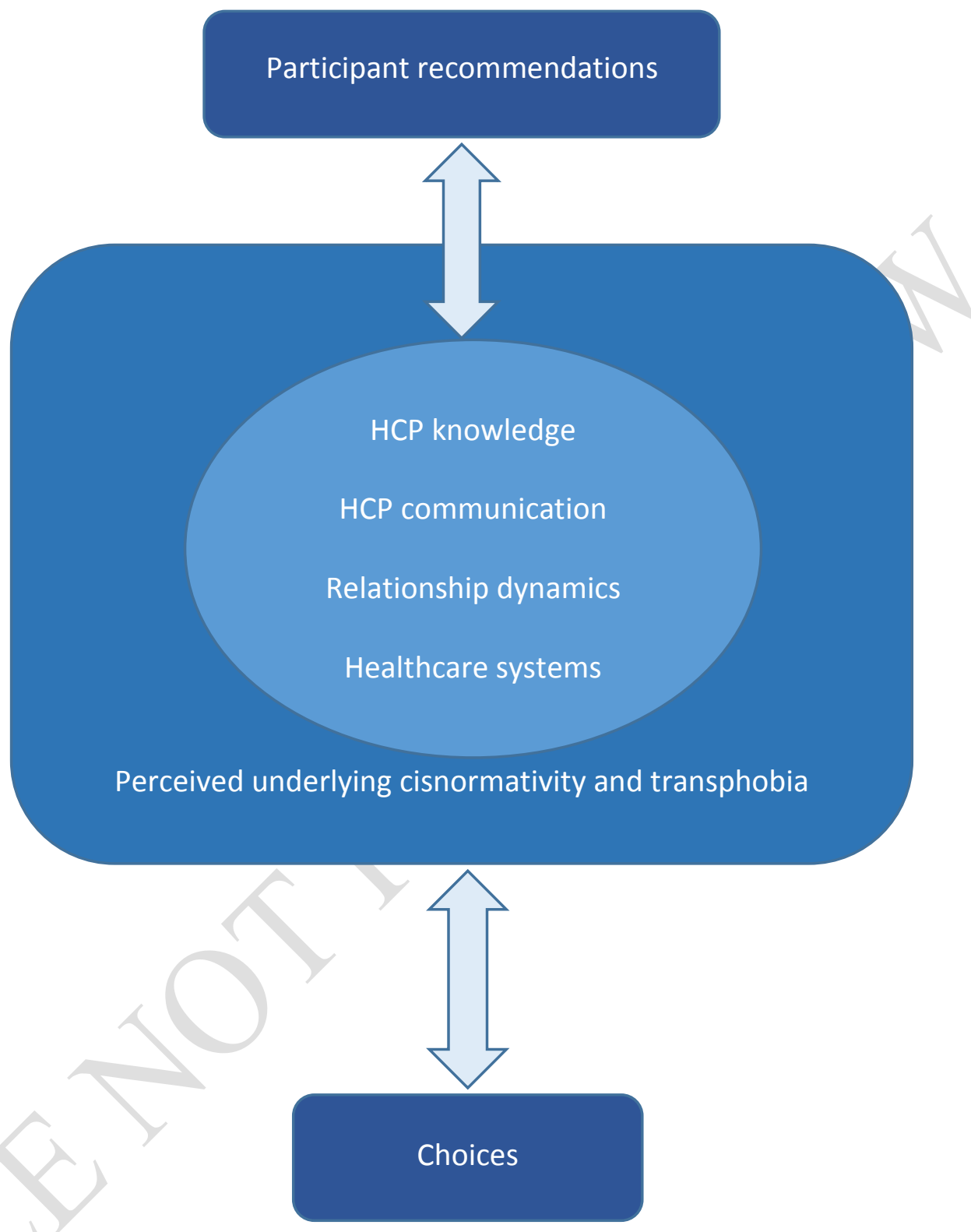

Notes: This figure depicts the relationship between the four main themes, underlying subtheme, and peripheral themes. The centre oval represents the four main themes related to participant healthcare experiences. The shaded oblong underlying this represents the perceived cisnormativity and transphobia subtheme underlying the four main themes. The darker-shaded peripheral oblongs represent the two peripheral themes of choices and participant recommendations. Participant recommendations were influenced by and directed towards the four main themes, while the choices participants made about their healthcare depended on their response to the perceived cisnormativity and transphobia underlying the four main themes. 


\section{Appendix}

\section{Search Strategy by Database}

\section{MEDLINE}

The following Medical Subject Headings (MeSH) terms and keywords were used as search terms:

- Transgender:

o MeSH: Transgender Persons (exp); Transsexualism; Health Services for Transgender Persons.

o Keywords: transgender; trans-gender; trans gender; transgend*; transsex* .

- Perspectives:

o MeSH: Emotions; Life Change Events; Attitude; Attitude to Health (exp).

o Keywords: perception*; thought*; feeling*; viewpoint*; opinion*; perspective*; attitude*; experience*; belief*.

- Healthcare:

o MeSH: Health Care (non mesh); Comprehensive Health Care; Delivery of Health Care (exp); Health Personnel; Primary Health Care; Health Services.

o Keywords: health care; health-care; healthcare.

The search terms of each concept were combined with the Boolean operator "OR.

Concepts were then linked with the “AND” Boolean operator to create a search of: (transgender search terms) AND (perspectives search terms) AND (healthcare search terms). The results of the combined concept search were then limited to English language and humans. This produced 889 results.

\section{CINAHL}

The following subject headings and keywords were used as search terms: 
- Transgender:

o Subject headings: Transgender Persons (+); Transsexualism; Health Services for Transgender Persons.

o Keywords: transgender; trans-gender; trans gender; transgend*; transsex* .

- Perspectives:

o Subject headings: Attitude; Public Opinion; Social Attitudes; Life Experiences; Health Beliefs; Attitude to Health; Attitude to Medical Treatment; Emotions; Life Change Events.

o Keywords: perception*; thought*; feeling*; viewpoint*; opinion*; perspective*; attitude*; experience*; belief*.

- Healthcare:

o Subject headings: Health Care Delivery (+); Health Personnel; Primary Health Care; Health Services; Health Services Needs and Demand.

o Keywords: health care; health-care; healthcare.

The search terms of each concept were combined with the Boolean operator "OR. Concepts were then linked with the “AND” Boolean operator. The limiters of English Language, Peer Reviewed, and Human were then applied to the results of the combined concept search to produce 175 results.

\section{PsycINFO}

The following subject headings and keywords were used as search terms:

- Transgender:

o Subject headings: Transgender (explode); Transsexualism.

o Keywords: transgender; trans-gender; trans gender; transgend*; transsex*; health services for transgender persons. 
- Perspectives:

o Subject headings: Social Perception; Experiences (Events); Attitudes; Perception; Emotions; Life Experiences; Public Opinion; Stereotyped Attitudes; Implicit Attitudes; Explicit Attitudes; Community Attitudes; Client Attitudes; Life Changes; Health Attitudes.

o Keywords: perception*; thought*; feeling*; viewpoint*; opinion*; perspective*; experience*; belief*.

- Healthcare:

o Subject headings: Health Care Services; Health Care Seeking Behavior; Health Care Utilization; Primary Health Care; Health Care Delivery (explode); Health Personnel; Allied Health Personnel.

o Keywords: health care; health-care; healthcare; comprehensive health care.

The search terms of each concept were combined with the Boolean operator "OR. Concepts were then linked with the “AND” Boolean operator. The combined concept search results were then limited to English language, human population, and peer reviewed. This resulted in 651 results.

\section{Informit, Scopus and Web of Science (Non-Subject Heading Databases)}

As these databases did not utilise subject headings, following keywords were used as search terms:

- Transgender:

o Keywords: transgender persons; transgender; trans-gender; trans gender; transgend*; transsexualism; transsex*; health services for transgender persons.

- Perspectives: 
o Keywords: perception*; thought*; feeling*; viewpoint*; opinion*; public opinion; perspective*; experience*; life experiences; belief*; attitude; social attitudes; attitude to health; attitude to medical treatment; emotions; life change events.

- Healthcare:

o Keywords: health care; health-care; healthcare; comprehensive health care; delivery of health care; health personnel; primary health care; health services; health services needs and demand.

The search terms of each concept were combined with the Boolean operator "OR. Concepts were then linked with the “AND” Boolean operator. In Informit, these terms were searched in "All Fields.” This produced 31 results. There were no options to limit the search. In Scopus, these keywords were searched in “Article title, Abstract, Keywords.” The combined concept search results were then limited to English. This resulted in 1067 results. In Web of Science, the combined concept search results were limited to the English language. This resulted in 509 results. 